\title{
Conditioned medium from stimulated mononuclear leucocytes potentiates the ability of human neutrophils to damage human articular cartilage
}

\author{
EDNA J BATES, INGEBORG C KOWANKO, AND ANTONIO FERRANTE \\ From the University Department of Paediatrics and the Department of Immunology, Adelaide Children's \\ Hospital, South Australia
}

SUMmary Human neutrophils were able to degrade proteoglycan and inhibit its synthesis when incubated with human articular cartilage coated with heat aggregated immunoglobulin $G$. These effects were potentiated when culture medium conditioned by mononuclear leucocytes stimulated with killed Staphylococcus aureus was also present during the incubations. Neutrophils preincubated with this conditioned medium and washed before incubation with cartilage also showed an increased ability to degrade proteoglycan and inhibit its synthesis. The percentage of neutrophils binding to cartilage was significantly increased in the presence of this conditioned medium.

Key words: polymorphonuclear leucocytes, cartilage injury, chondrocyte impairment, proteoglycan degradation, proteoglycan synthesis, cytokines, neutrophil respiratory burst, neutrophil degranulation.

In rheumatoid arthritis a progressive destruction of cartilage occurs which has been attributed traditionally to enzymatic degradation ${ }^{1}$ and more recently to a decreased ability of the tissue to synthesise its matrix components. ${ }^{2-4}$ During the acute phase of both the human disease and in animal models and during acute exacerbations of the disease vastly increased numbers of inflammatory cells, mostly neutrophils, are seen in the joint and synovial tissue. ${ }^{56}$ It has also been shown that neutrophils invade cartilage in chronically inflamed joints, especially at the cartilage-pannus border. ${ }^{78}$ These neutrophils have been implicated in the degradation and impaired metabolism of cartilage through their release of lysosomal enzymes, particularly elastase, ${ }^{8-13}$ and oxygen metabolites. ${ }^{14-17}$

The rheumatoid joint is characterised by proliferation of synoviocytes and accumulation of neutrophils, macrophages, plasma cells, and $T$ and $B$ lymphocytes. These cells produce a family of mono-

Accepted for publication 15 June 1987.

Correspondence to Dr Edna J Bates, Department of Paediatrics, Adelaide Children's Hospital, North Adelaide, South Australia 5006 . kines and lymphokines (cytokines), of which a number, including interleukin $1,{ }^{18}$ interleukin $2,{ }^{19}$ interferons, ${ }^{2021} \mathrm{~B}$ cell colony stimulating factor, ${ }^{22}$ and macrophage migration inhibitory factor, ${ }^{23}$ have been identified in the synovial fluid of patients with rheumatoid arthritis. Neutrophil function relevant to tissue damage can be modified by a number of cytokines ${ }^{24-31}$ and it is likely that this contributes to neutrophil mediated damage of cartilage in rheumatoid arthritis.

The objective of this study was to investigate whether neutrophils treated with cytokine-rich conditioned medium show an increased ability to damage cartilage in culture. For these experiments cells and tissue of human origin were used.

\section{Materials and methods}

PREPARATION AND CULTURE OF ARTICULAR C A R T I L A G E

Articular cartilage slices (4-10 g) were removed at necropsy (within 24 hours of death) from the nonarthritic knees of adult humans (aged 18-50 years), cut into $2-3 \mathrm{~mm}$ square pieces, and cultured in $60 \mathrm{ml}$ 
culture medium for three to five days before use. Culture medium was replaced daily and consisted of Dulbecco's modified Eagle's medium (Flow Laboratories, Sydney, Australia) containing $5 \%$ heat inactivated $\left(56^{\circ} \mathrm{C} / 30 \mathrm{~min}\right)$ human $\mathrm{AB}$ serum (Flow Laboratories, Sydney, Australia). Cultures were maintained at $37^{\circ} \mathrm{C}$ in an atmosphere of $5 \% \mathrm{CO}_{2}$ and high humidity.

\section{PREPARATION OF LEUCOCYTES}

Mononuclear leucocytes (MNL) and neutrophils were prepared from blood of healthy volunteers by a rapid single step procedure. ${ }^{32}{ }^{33}$ Heparinised blood $(6 \mathrm{ml})$ was layered onto Ficoll-Hypaque $(4 \mathrm{ml}$; density $1 \cdot 114$ ) and centrifuged at 400 gav for $30-40$ min at room temperature. The leucocytes resolved into two distinct bands. Each band was washed three times. The upper band containing MNL was washed in medium 199 (Flow Laboratories, Sydney, Australia), and the lower band containing neutrophils was washed in Hanks's balanced salt solution without phenol red (HBSS). Neutrophils were finally resuspended at a concentration of $4 \times 10^{7}$ cells $/ \mathrm{ml}$ HBSS. Preparations of neutrophils were $98-99 \%$ pure and $>99 \%$ viable as judged by the ability to exclude trypan blue. Neutrophils were kept at room temperature and used within $30 \mathrm{~min}$ of preparation.

PREPARATION OF MEDIA CONDITIONED BY $M N$ L

Media conditioned by MNL were prepared as described previously. ${ }^{30}$ MNL were cultured in RPMI 1640 medium (Flow Laboratories, Sydney, Australia) containing $2.5 \%$ heat inactivated AB serum for 72 hours at $37^{\circ} \mathrm{C}$ with heat killed, formalin fixed Staphylococcus aureus.

Filtered medium conditioned by $S$ aureus treated MNL was termed 'stimulated conditioned medium' (sCM), medium cultured in the absence of MNL or bacteria was referred to as 'non-conditioned medium' (NCM), and medium cultured with bacteria but without MNL was termed BCM.

TREATMENT OF CARTILAGE WITH NEUTROPHILS

On the day of experiment cartilage was coated with heat aggregated human immunoglobulin $G$ (HAGG). This was prepared by heating human immunoglobin G (CSL Laboratories, Melbourne, Australia) at $63^{\circ} \mathrm{C}$ for one hour at a concentration of $20 \mathrm{mg} / \mathrm{ml}$ in phosphate buffered saline, $\mathrm{pH} \mathrm{7 \cdot 2}$. The cartilage was incubated with HAGG at $5 \mathrm{mg} / 100 \mathrm{mg}$ tissue for one hour at $37^{\circ} \mathrm{C}$ in the presence of an equal volume of culture medium. The tissue was washed three times with HBSS, and 50-150 mg wet weight of tissue was distributed into sterile poly- styrene centrifuge tubes (Bunzl, South Australia) containing $1 \mathrm{ml}$ HBSS

Cartilage was then incubated for 2.5 hours at $37^{\circ} \mathrm{C}$ in a total volume of $2 \mathrm{ml}$ HBSS. These incubationso contained either neutrophils that had been preincu bated with conditioned media $(\mathrm{sCM}, \mathrm{NCM}$, or $\mathrm{BCM}$ ) or untreated neutrophils and conditione $\mathrm{C}^{\mathbb{D}}$ media (sCM or NCM) added together to the tissue For preincubation of neutrophils, one part neutro- phils (at $4 \times 10^{7}$ cells $/ \mathrm{ml}^{\mathrm{HBSS}}$ ) was incubated with three parts conditioned medium (diluted 1:2 with $\vec{\omega}$ HBSS) for one hour. After centrifugation (5 min $\mathrm{S}$ $400 \mathrm{gav})$ the cells were counted and resuspended at $5 \times 10^{6} / \mathrm{ml} \mathrm{HBSS}$ and $1 \mathrm{ml}$ added to the cartilage Incubations without neutrophils contained $1 \mathrm{mF}$ HBSS instead of cells.

Incubations of cartilage with untreated neutro-o phils contained $0.25 \mathrm{ml}$ cells $\left(7 \times 10^{6}\right.$ cells $)$ and 0.75 $\mathrm{ml}$ conditioned media. Incubations without neutrophils contained $0.25 \mathrm{ml}$ HBSS instead of cells.

After incubation the medium was removed (see 'Proteoglycan degradation') and the tissue washed with $1 \mathrm{ml}$ of serum free culture medium. The tissuee was either used to measure day 0 proteoglycan ${ }^{\circ}$ degradation or synthesis or cultured with $4 \mathrm{mlO}$ culture medium for a further two days, the mediums being replaced daily. Proteoglycan degradation or synthesis was measured on both of these days.

PROTEOGLYCAN SYNTHESIS

Proteoglycan synthesis was measured by the incorporation of sodium $\left[{ }^{35} \mathrm{~S}\right]$ sulphate (Radiochemical Centre, Amersham, UK) into glycosaminoglycanon chains. Cartilage was incubated with $2 \mathrm{ml}$ culture medium supplemented with $\left[{ }^{35} \mathrm{~S}\right]$ sulphate $\left(1 \cdot 11 \frac{2}{2}\right.$ $\mathrm{MBq}$ /incubation, final specific activity $0.74 \mathrm{MBq}$ 윽 $\mu \mathrm{mol})$ for two hours. The ${ }^{35} \mathrm{~S}$ labelled glycosaminoglycans in the tissue were extracted with $2 \mathrm{ml} 0.5 \mathrm{MO}$ $\mathrm{NaOH}$ for 48 hours at room temperature and $0.25 \mathrm{ml}$ aliquots applied to Sephadex G-25 PD-10옹 columns (Pharmacia AB, Uppsala, Sweden) and the $>$ eluent counted for radioactivity. ${ }^{14}$

PROTEOGLYCAN DEGRADATION

Proteoglycan degradation was measured by deter- $N$ mining the release of $\left[{ }^{35} \mathrm{~S}\right]$ sulphate labelled proteo- $-\omega$ glycan from cartilage. Cartilage was labelled for 20 hours with sodium $\left[{ }^{35} \mathrm{~S}\right]$ sulphate $(3.7 \mathrm{MBq} / \mathrm{g}$ tissue $)$ 읃 at a concentration of $1 \cdot 11 \mathrm{MBq} / \mathrm{ml}$ culture medium $\Phi$ (final specific activity $1.48 \mathrm{MBq} / \mu \mathrm{mol}$ ) before use in ${ }^{+}$ experiments. The tissue was washed extensively with serum free culture medium to remove excess label before incubation with $\mathrm{HAGG}$ as described above.

After incubation of cartilage with neutrophils the washed tissue was extracted with $2 \mathrm{ml} 0.5 \mathrm{M} \mathrm{NaOHo}$ 
for 48 hours. Cartilage incubation media were centrifuged to remove neutrophils, and $250 \mu \mathrm{l}$ aliquots were applied to Sephadex G-25 PD-10 columns and the eluent counted for radioactivity. $\mathrm{NaOH}$ extracts were similarly eluted and counted. Proteoglycan release during cartilage incubation with neutrophils (day 0 ) was calculated from these counts. For calculation of day 1 release the subsequent 24 hour culture media and corresponding $\mathrm{NaOH}$ extracts of tissue were analysed on Sephadex G-25. Similar analyses were performed for day 2 release.

BINDING OF NEUTROPHILS TO CARTILAGE Neutrophils were labelled with ${ }^{51} \mathrm{Cr}$ by incubating $1 \times 10^{7}$ cells with $7 \cdot 4 \mathrm{MBq}$ of sodium $\left[{ }^{51} \mathrm{Cr}\right]$ chromate (16.7 GBq/mg Cr; Radiochemical Centre, Amersham, UK) at $37^{\circ} \mathrm{C}$ for one hour. The cells were then washed five times with serum free culture medium, once with HBSS, and resuspended at $4 \times 10^{7}$ cells $/ \mathrm{ml}$ HBSS. The cells $\left(1 \times 10^{7}\right)$ were incubated for $2 \cdot 5$ hours with HAGG coated cartilage in the presence of NCM or sCM as described above.

After incubation the cartilage was washed with serum free culture medium and extracted with $2 \mathrm{ml}$ $0.5 \mathrm{M} \mathrm{NaOH}$ for 48 hours. A sample of neutrophils was centrifuged and the pellet extracted with $2 \mathrm{ml}$ $0.5 \mathrm{M} \mathrm{NaOH}$. These extracts were counted in a gammacounter.

EXPRESSION OF RESULTS AND STATISTICAL ANALYSIS

The results presented are from one or more experiments. Each experiment was repeated three times with different batches of cartilage and neutrophils. The large variation in both cartilage metabolism and its response to neutrophil treatment precluded combining the data from all three experiments. The

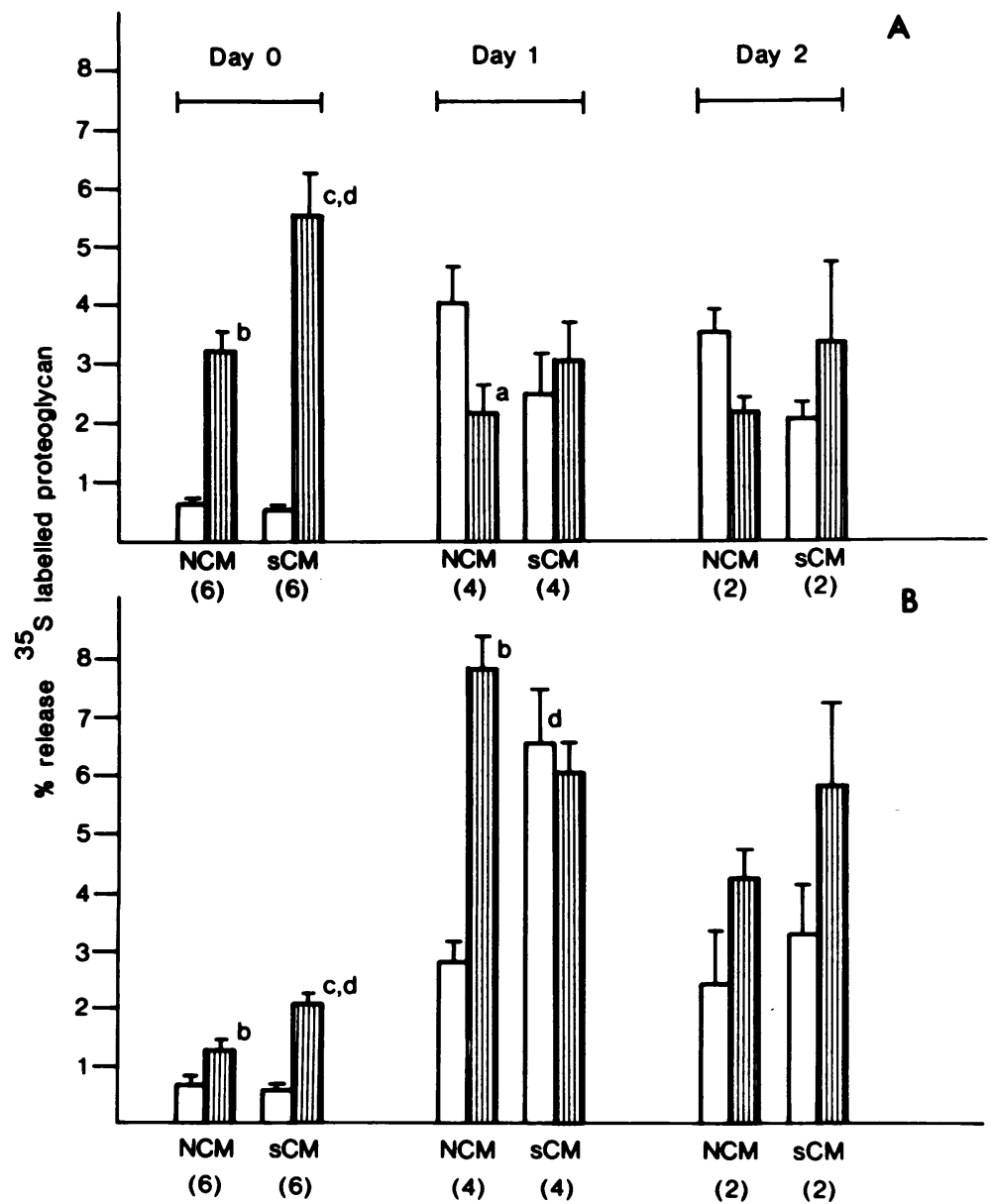

Fig. 1 Proteoglycan degradation during treatment of cartilage with neutrophils in the presence of 'nonconditioned medium' (NCM) or 'stimulated conditioned medium' (sCM). Open and striped bars indicate incubations of cartilage in the absence and presence of neutrophils respectively. $A$ and $B$ represent results from separate experiments. Values are expressed as mean (SEM) except where $n=2$ (mean \pm range of values). ' $a$ ' and ' $b$ ' indicate $p<0.02 ; 0.01$ for the effect of neutrophils in the presence of NCM, 'c' indicates $p<0.01$ for the effect of neutrophils in the presence of $s C M$, and ' $d$ ' indicates $p<0.02$ for the effect of neutrophils plus $s C M$ compared with neutrophils plus NCM. 
responses observed were always present, however, and two typical experiments are generally presented to illustrate the large variation between experiments. Statistical significance was analysed by the two tailed $t$ test for unpaired data.

\section{Results}

Preliminary experiments showed that neutrophils were unable to cause proteoglycan degradation when incubated with cartilage that had not been coated with HAGG (percentage release ${ }^{35} \mathrm{~S}$ labelled proteoglycan on day 0 was $3.3(0.5)$ and $3.3(0.4)$, mean (SEM) for six cultures in the absence and presence of neutrophils). When neutrophils were incubated with cartilage coated with HAGG, however, an increased degradation of proteoglycan always resulted on day 0 (Fig. 1). Proteoglycan degradation was further increased by $60-75 \%$ if $\mathrm{sCM}$ was also present during these incubations. The sCM itself, in the absence of cells, did not cause degradation of proteoglycan (Fig. 1). A neutrophil stimulation of? degradation in the presence of NCM or SCM was not consistently seen on day 1 or day 2 (Fig. 1).

When proteoglycan synthesis was measured neu trophils did not alter synthesis on day 0 in the presence of NCM or SCM (Fig. 2). In one individua by day 1 , however, incubation with neutrophils in the presence of NCM caused a $65 \%$ inhibition of proteoglycan synthesis (Fig. 2B). By day 2 , incuba $-\overrightarrow{0}$ tion with neutrophils in the presence of NCM causeda $45-83 \%$ inhibition of proteoglycan synthesis. $\vec{\omega}$ When sCM was present a $55-60 \%$ inhibition of synthesis was seen on day 2 (Fig. 2). Under theseo conditions the $\mathrm{sCM}$ itself, in the absence of cells $\stackrel{\rightarrow}{\rightarrow}$ caused an $84-87 \%$ inhibition of proteoglycan syn thesis. This inhibition by the $\mathrm{SCM}$ was already apparent by day 1 (Fig. 2).

Further experiments were performed with neutrophils which had been preincubated with conditioned media and washed before incubation with cartilage The results presented in Fig. 3 show that neutrophils

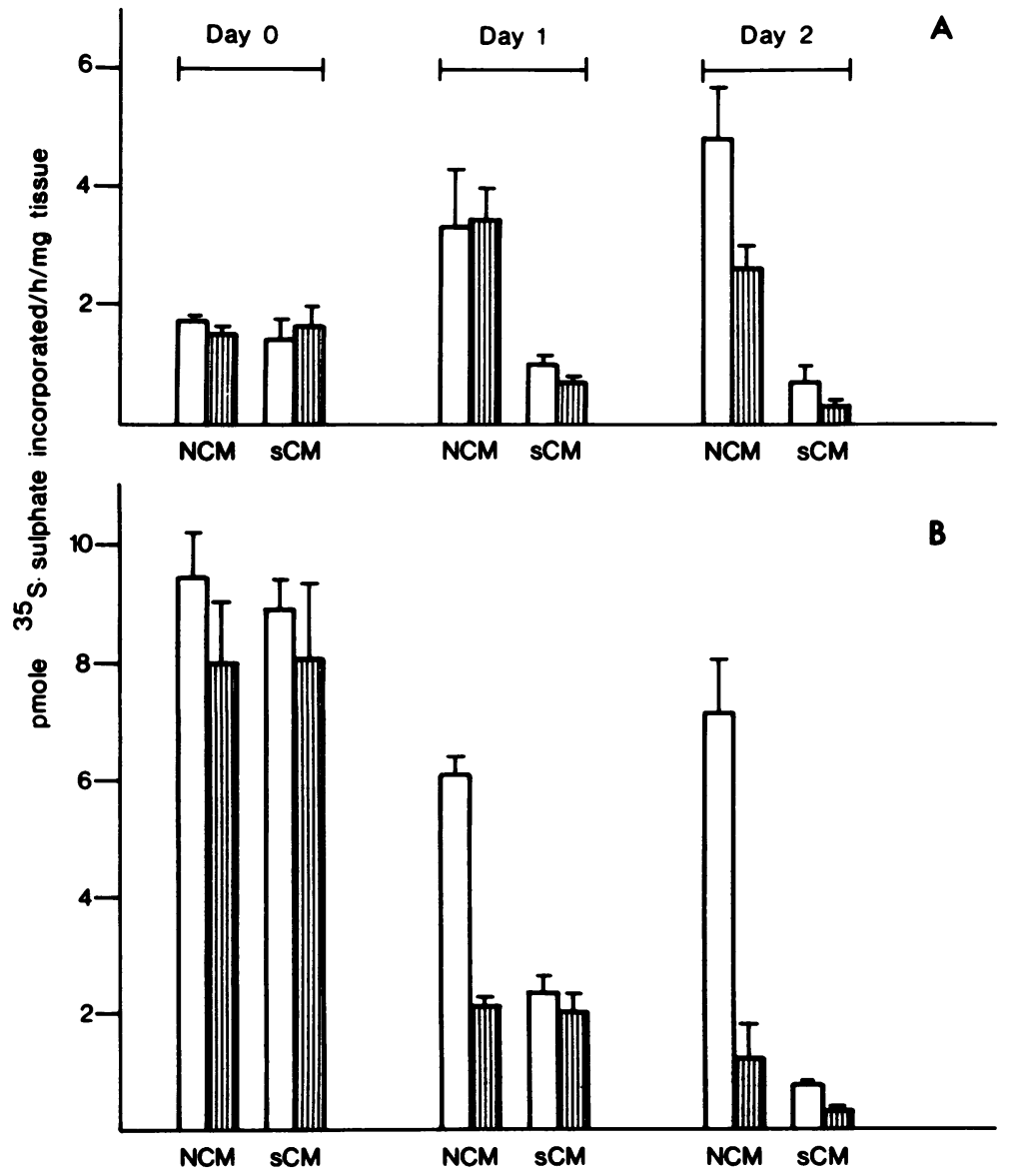

Fig. 2 Proteoglycan synthesis after treatment of cartilage with neutrophils in the presence of NCM or SCM. Open and striped bars indicate incubations of cartilage in the absence and presence of neutrophils respectively. $A$ and $B$ represent results from separate experiments Values are expressed as mean \pm range of values for duplicate cultures. 
preincubated with sCM caused almost four- to fivefold more degradation on day 0 than cells preincubated with NCM. This potentiation of degradation by neutrophils treated with $\mathrm{sCM}$ was reduced by day 1 and day 2 (Fig. 3). A neutrophil stimulation of degradation by neutrophils treated with NCM was not apparent after day 0 (Fig. 3).

The results presented in Fig. 4 show that neutrophils preincubated with NCM could inhibit synthesis by day 1 and day 2 . On day 1 (Fig. 4B) and day 2 (Figs 4A and 4B) this inhibition was considerably increased by preincubating neutrophils with $\mathrm{sCM}$.
The results seen with sCM stimulated neutrophils could have been due to a bacterial contaminant in the sCM. Medium (BCM) generated by incubating $S$ aureus in the absence of MNL was therefore compared with NCM and SCM for its effect on neutrophil mediated cartilage damage. Neutrophils preincubated with $\mathrm{BCM}$ gave an identical inhibition of proteoglycan synthesis to that by neutrophils preincubated with NCM (Fig. 4B). Neutrophils preincubated with NCM or BCM also caused similar levels of proteoglycan degradation (Fig. 5), thus demonstrating that a bacterial contaminant was not
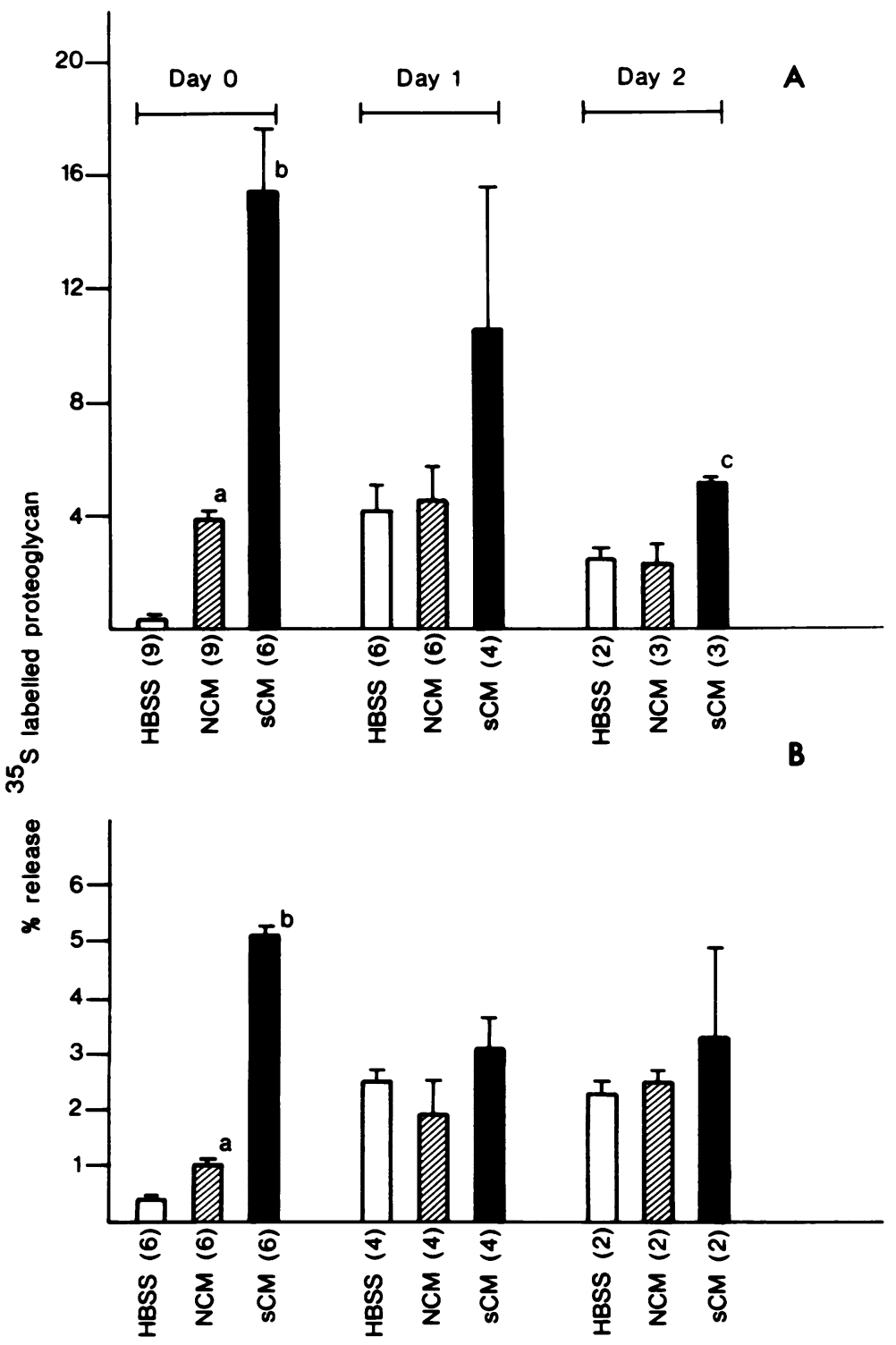

Fig. 3 Effect of neutrophils preincubated with NCM or SCM on proteoglycan degradation. Open bars indicate incubations without neutrophils. Hatched and closed bars indicate incubations with neutrophils preincubated with NCM or $s C M$ respectively. $A$ and $B$ represent results from separate experiments. Values are expressed as mean (SEM) except where $n=2$ (mean \pm range of values). Numbers in parentheses indicate the number of cultures. ' $a$ ' indicates $p<0.01$ for the effect of neutrophils preincubated with $N C M$, ' $b$ ' and ' $c$ ' indicate $p<0.01$ and $p<0.02$ respectively for the effect of neutrophils plus sCM compared with neutrophils plus NCM. 


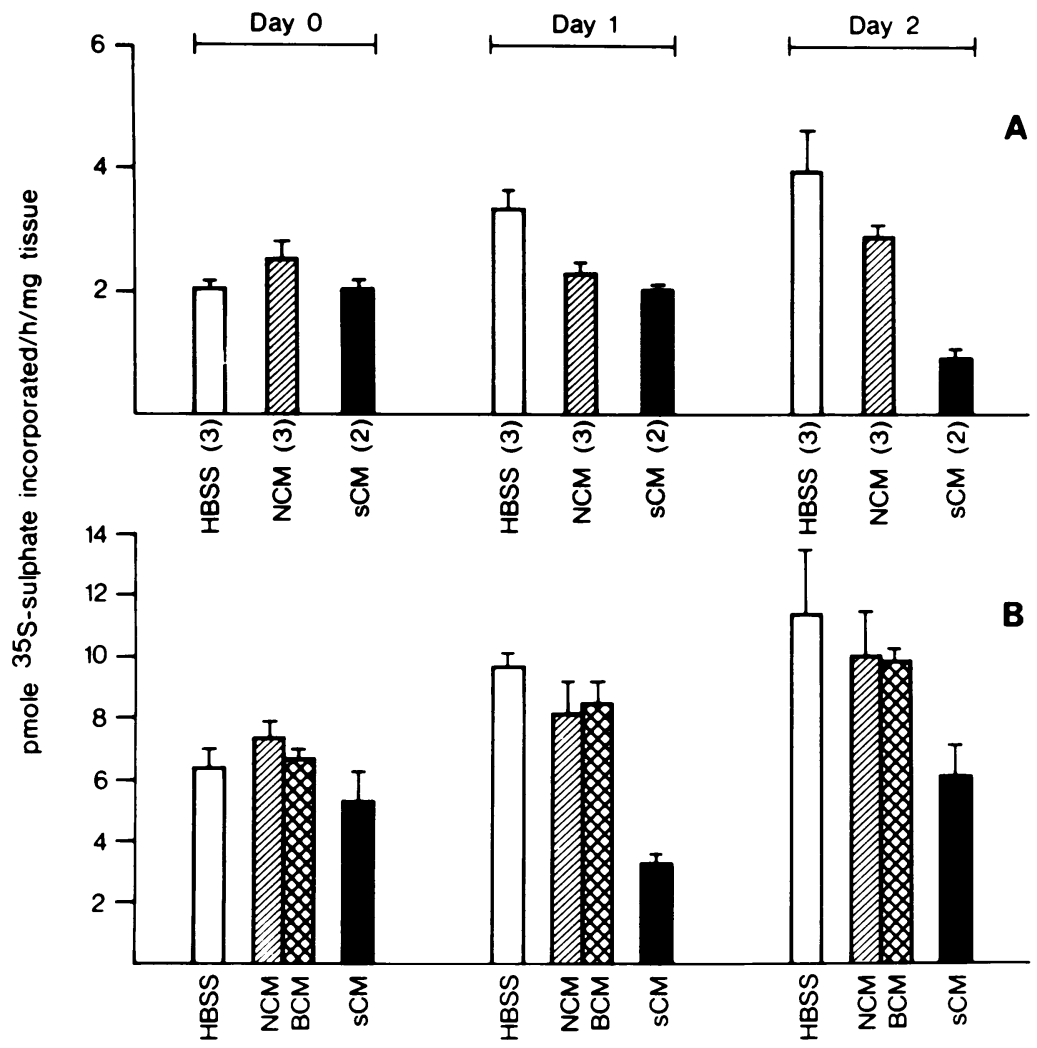

Fig. 4 Effect of neutrophils preincubated with $N C M / B C M$ or sCM on proteoglycan synthesis. Open bars indicate incubations without neutrophils. Hatched and closed bars indicate incubations with neutrophils preincubated with. $N C M / B C M$ or $S C M$ respectively. $A$ and $B$ represent results from separate experiments. Values are expressed as mean (SEM) except where $n=2$ (mean \pm range of values). Numbers in parentheses indicate the numbers of cultures. Throughout experiment $B$ duplicate cultures were used.

responsible for the effect of $\mathrm{sCM}$ on neutrophil mediated cartilage damage.

Using ${ }^{51} \mathrm{Cr}$ labelled neutrophils it was found that about $8 \%$ of the neutrophils adhered to cartilage in the presence of NCM (Table 1). This percentage was significantly increased in the presence of $\mathrm{sCM}$ (Table 1). When the data from three experiments were combined the percentage of neutrophils bound was increased from $9.8(1.7)$ to $18.0(3.9)$ (mean

Table 1 Binding of neutrophils to cartilage

\begin{tabular}{lc}
\hline Addition & $\%$ Neutrophils bound \\
\hline $\mathrm{NCM}$ & $7 \cdot 8(1 \cdot 8)$ \\
$\mathrm{SCM}$ & $25 \cdot()(1 \cdot 2)^{*}$ \\
\hline
\end{tabular}

${ }^{51} \mathrm{Cr}$ labelled neutrophils were incubated with cartilage in the presence of NCM or SCM. Results are calculated by expressing the ${ }^{51} \mathrm{Cr}$ bound to the cartilage as a percentage of that added to the incubations as ${ }^{51} \mathrm{Cr}$ labelled neutrophils. Results are expressed as mean (SEM) of four cultures.

${ }^{*} \mathrm{p}<0.001$ for the binding of neutrophils in the presence of $\mathrm{sCM}$ compared with NCM
(SEM)) for neutrophils incubated in the presence of $\mathrm{NCM}$ and $\mathrm{sCM}$ respectively.

\section{Discussion}

Extensive loss of proteoglycan has been described in cartilage biopsy specimens taken from rheumatoido arthritic patients as early as 18 days after onset of symptoms. ${ }^{34}$ This may occur either by an acceler-O을. ated breakdown of proteoglycan or by an inhibition of its renewal through loss by normal turnover. Inflammatory cells such as neutrophils and macro- $N$ phages are believed to have an important role in N these events. In vitro, neutrophils attach in largeo numbers to rheumatoid articular cartilage comparedo with normal cartilage. ${ }^{35}$ The neutrophils invade the tissue and phagocytose amorphous material prob- $\stackrel{\oplus}{+}$ ably containing immune complexes. ${ }^{35}$ Our results 0 indicate that human neutrophils are capable of causing damage to human cartilage in culture. This damage involved both enhanced proteoglycan $\bar{\Phi}$ degradation and impaired proteoglycan synthesis.

Damage was only seen if the cartilage was coated with HAGG. The use of cartilage coated witho 


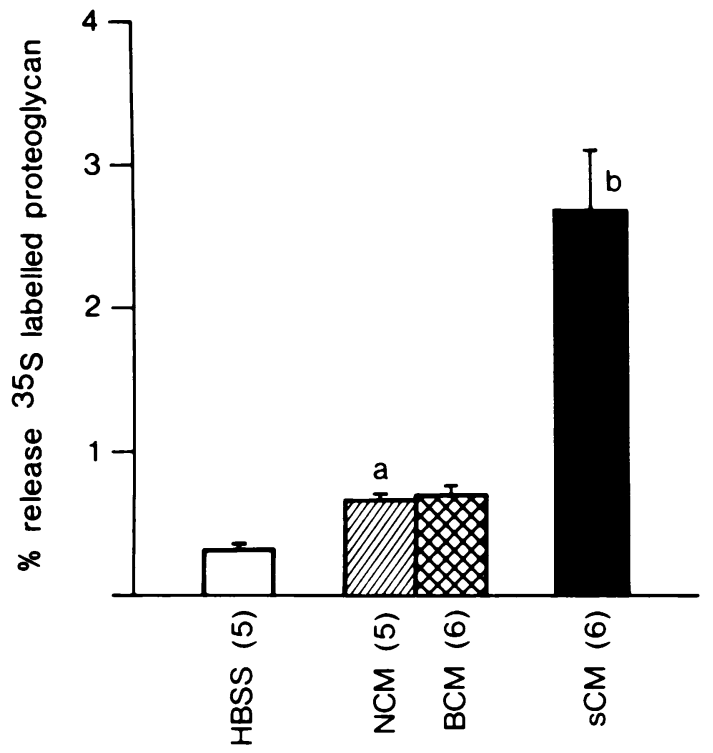

Fig. 5 Proteoglycan degradation during treatment of cartilage with neutrophils preincubated with NCM/BCM or sCM. Open bars indicate incubations without neutrophils. Hatched and closed bars indicate incubations with neutrophils preincubated with NCM/BCM or $S C M$ respectively. Values are expressed as mean (SEM).

Numbers in parentheses indicate the number of cultures. ' $a$ ' indicates $p<0.01$ for the effect of neutrophils preincubated with NCM, ' $b$ ' indicates $p<0.01$ for the effect of neutrophils plus sCM compared with neutrophils plus NCM.

HAGG was designed to mimic the in vivo situation where immune complexes are found in collagenous joint tissues of naturally occurring and experimental arthritis. ${ }^{36} 37$ There is in vitro evidence that both HAGG and immune complexes activate neutrophils directly, ${ }^{38}$ and cartilage coated with HAGG has been successfully used to assess the effects of clinically useful drugs on enzyme release by human neutrophils in relation to the degradation of rabbit cartilage. $^{12}$

Neutrophil mediated cartilage damage was enhanced by SCM. This is consistent with our previous findings that $\mathrm{sCM}$ potentiates neutrophil damage of large non-phagocytosable organisms such as free-living amoebae. ${ }^{2829} 3139$ The ability of sCM to potentiate the effects of neutrophils on cartilage is relevant in vivo in rheumatoid arthritis. Synovial fluid from inflamed joints contains cytokines, ${ }^{18-23}$ which are likely to be present in the SCM used in our experiments. Preliminary results have shown that sCM contains interleukin 1 and colony stimulating factor activities (unpublished results). Recently we have shown that a neutrophil stimulatory activity is present, which is unlikely to be one of the previously described cytokines. ${ }^{30}$

The impaired synthesis and increased degradation of cartilage seen after neutrophil treatment could result from a number of neutrophil functions. The ability of neutrophils to release granule enzymes capable of tissue damage is well documented. ${ }^{12} 40-42$ In vivo cartilage is bathed in synovial fluid containing antiproteinases capable of inactivating proteinases released from neutrophils. ${ }^{43}$ When large substances such as entrapped immune complexes in cartilage are phagocytosed, however, neutrophils show 'frustrated phagocytosis' ${ }^{40}$ during which the close contact between neutrophil and cartilage excludes synovial fluid from this microenvironment. Thus degradation of cartilage in vivo by neutrophils is certainly feasible and it is of interest that neutrophils have been shown to cause proteolysis of a fibronectin substrate bound onto microtitre plates even in the presence of inhibitors. ${ }^{42}$ In addition, neutrophil elastase has been found in the superficial layer of proteoglycan depleted, pannus free rheumatoid articular cartilage, ${ }^{9}$ whereas no elastase activity could be detected in the synovial fluid from patients with rheumatoid arthritis. ${ }^{9}$ Neutrophil elastase has also been shown to inhibit the synthesis of proteoglycan by bovine articular cartilage in culture. ${ }^{13}$ Thus neutrophil proteinases may be important both in the degradation and impaired synthesis of proteoglycan that we have found. Inclusion of soybean trypsin inhibitor, known to inhibit neutrophil serine proteinases, ${ }^{44}{ }^{45}$ during incubation of cartilage with neutrophils did not alter degradation or impairment of synthesis, thus suggesting that frustrated phagocytosis is operative in our experimental system (results not shown). The fact that neutrophils are able to kill cells and organisms larger than themselves, such as amoebae and tumour cells, ${ }^{28} 2939$ through frustrated phagocytosis adds weight to the idea that such a mechanism is operative in cartilage damage.

Oxygen metabolites released during the neutrophil respiratory burst ${ }^{46}$ have been shown to cause an inhibition of proteoglycan synthesis. ${ }^{14} 15$ Although these species can cause the breakdown of hyaluronic acid, ${ }^{16}$ they have little effect on the proteoglycan monomer. ${ }^{47}$ Recently there has been one report that repeated doses of oxygen metabolites can cause proteoglycan release from cartilage. ${ }^{17}$ These authors also presented evidence that cartilage altered by prior elastase treatment was more susceptible to attack by oxygen metabolites, ${ }^{17}$ and it is likely that the combined action of a number of neutrophil functions is responsible for the results we see.

Neutrophil degradation was not mediated through 
activation of chondrocyte function as the same potentiation of degradation by neutrophils treated with $\mathrm{SCM}$ was seen when cartilage was killed by freezing (in liquid nitrogen) and thawing three times (results not shown).

The ability of the sCM to potentiate neutrophil damage to cartilage may result from a stimulation of neutrophil granule enzyme or oxygen metabolite release, or both. The neutrophil stimulatory activity we have shown to be present in the $\mathrm{sCM}^{30}$ caused a stimulation of both granule enzyme release and the respiratory burst and an inhibition of neutrophil locomotion. In the present experiments neutrophil binding to cartilage was increased by $\mathrm{SCM}$ and this may be important in the ability of sCM to potentiate neutrophil damage to cartilage.

Our results indicate that the $\mathrm{sCM}$ itself was capable of inhibiting proteoglycan synthesis after one or more days in culture. This was not unexpected as it is well documented that lymphokines and monokines are capable of inhibiting proteoglycan synthesis and causing proteoglycan degradation. ${ }^{48-53}$ There was, however, no consistent effect of sCM alone on degradation over the same time period.

Although neutrophils have been implicated in the degradation and impaired metabolism of cartilage in rheumatoid arthritis, ${ }^{8-17}$ this is, to our knowledge, the first report where both increased degradation and a decreased synthesis of proteoglycan have been shown using human neutrophils and human cartilage in culture. The ability of cytokine-rich $\mathrm{sCM}$ to potentiate this damage suggests an important role for neutrophil mediated damage in rheumatoid arthritis.

We are grateful to Dr L P Bignold of the Department of Pathology, University of Adelaide for obtaining necropsy material, D P Harvey for excellent technical assistance, and J Raphael for typing the manuscript. This work was supported by grants from the National Health and Medical Research Council of Australia, the Arthritis Foundation of Australia, and the Channel 10 Children's Medical Research Foundation.

\section{References}

1 Sheppard H. An update on mechanisms of cartilage destruction in rheumatoid arthritis. Aust NZ J Med 1983; 13: 195-200.

2 Lowther D A, Gillard G C. Carrageenin-induced arthritis. I. The effect of intra-articular carrageenin on the chemical composition of articular cartilage. Arthritis Rheum 1976; 19: 769-76.

3 Sandy J D, Lowther D A, Brown H L G. Antigen-induced arthritis: studies on the inhibition of proteoglycan systhesis observed in articular cartilage during short-term joint inflammation. Arthritis Rheum 1980; 23: 433-47.

4 Gillard G C, Lowther D A. Carrageenin-induced arthritis. II. Effect of intra-articular injection of carrageenin on the synthesis of proteoglycan in articular cartilage. Arthritis Rheum 1976; 19: 918-22.
5 Santer V, Sriratana A, Lowther D A. Carrageenin-induced $\stackrel{5}{\text {. }}$ arthritis. V. A morphologic study of the development of $\vec{\Rightarrow}$ inflammation in acute arthritis. Semin Arthritis Rheum 1983; 13: 160-8.

6 Zvaifler $\mathrm{N} \mathrm{J}$. The immunopathology of joint inflammation in rheumatoid arthritis. Adv Immunol 1973; 16: 265-336.

7 Mohr W, Westerhellweg H, Wessinghage D. Polymorpho- $\vec{\nabla}$ nuclear granulocytes in rheumatic tissue destruction. III. An $\varrho$ electron microscopic study of PMNs at the pannus-cartilage junction in rheumatoid arthritis. Ann Rheum Dis 1981; 40: 396-9.

8 Menninger $\mathrm{H}$, Putzier R, Mohr W, Wessinghage D, Tillman K. Granulocyte elastase at the site of cartilage erosion by rheuma- $\vec{\omega}$ toid synovial tissue. $Z$ Rheumatol 1980; 39: 145-56.

9 Velvart M, Fehr K, Baici A, et al. Degradation in vivo of articular cartilage in rheumatoid arthritis by leucocyte elastase from polymorphonuclear leucocytes. Rheumatol Int 1981; 1: 121-30.

10 Sandy J D, Sriratana A, Brown H L G, Lowther D A. Evidence for polymorphonuclear leucocyte-derived proteinases in arth-음 ritic cartilage. Biochem J 1981; 193: 193-202.

11 Janoff A, Feinstein G, Malemud C J, Elias J M. Degradation of cartilage proteoglycan by human leucocyte granule neutral proteases-a model of joint injury. I. Penetration of enzyme $\vec{乛}$ into rabbit articular cartilage and release of ${ }^{35} \mathrm{SO}_{4}$ labelled material from the tissue. J Clin Invest 1976; 56: 615-24.

12 Ignarro L J. Release of neutral protease and $\beta$-glucuronidase $\overrightarrow{0}$ from human neutrophils in the presence of cartilage treated $\infty$ with various immunologic reactants. J Immunol 1974; 113: 298-308.

13 Bartholemew J S, Lowther D A, Handley C J. Changes in proteoglycan biosynthesis following leukocyte elastase treatment of bovine articular cartilage in culture. Arthritis Rheum 1984; 27: 905-12.

14 Bates E J, Lowther D A, Handley C J. Oxygen free-radicals $\mathbb{D}$ mediate an inhibition of proteoglycan synthesis in cultured articular cartilage. Ann Rheum Dis 1984; 43: 462-9.

15 Bates E J, Johnson C C, Lowther D A. Inhibition of 3 proteoglycan synthesis by hydrogen peroxide in cultured bovine $?$ articular cartilage. Biochem Biophys Acta 1985; 838: 221-8.

16 Greenwald R A, Moy W W. Effect of oxygen-derived free radicals on hyaluronic acid. Arthritis Rheum 1980; 23: 455-63.

17 Burkhardt $\mathrm{H}$, Schwingel M, Menninger $\mathrm{H}$, Macartney $\mathrm{H}$ W, 응 Tschesche $\mathbf{H}$. Oxygen radicals as effectors of cartilage destruction. Direct degradative effect on matrix components and? indirect action via activation of latent collagenase from polymorphonuclear leukocytes. Arthritis Rheum 1986; 29: 379-87.

18 Wood D D, Ihrie E J, Dinarello C A, Cohen P L. Isolation of an interleukin-1-like factor from human joint effusions. Arth-J ritis Rheum 1983; 26: 975-83.

19 Wilkins J A, Warrington R J, Sigurdson S L, Rutherford W J. 음 The demonstration of an interleukin-2 like activity in the synovial fluids of rheumatoid arthritis patients. $J$ Rheumatolos 1983; 10: 109-13.

20 Degré $M$, Mellbye $O J$, Clarke-Jenssen $O$. Immune interferon $N$ in serum and synovial fluid in rheumatoid arthritis and related disorders. Ann Rheum Dis 1983; 42: 672-6.

21 Cesario T C, Andrews B S, Martin D A, et al. Interferon in synovial fluid and serum of patients with rheumatic disease. Rheumatol 1983; 10: 647-50.

22 Fay A C, Trudgett A, McCrea J D, et al. Detection and partial characterization of human B cell colony stimulating activity in $\square$ synovial fluids of patients with rheumatoid arthritis. Clin $\operatorname{Exp}$ Immunol 1985; 60: 316-22.

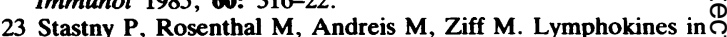
the rheumatoid joint. Arthritis Rheum 1975; 18: 237-43.

24 Smith R J, Bowman B J, Speziale S C. Interleukin-1 stimulates granule exocytosis from human neutrophils. Int J Immuno pharmacol 1986; 8: 33-40.

25 Berton G, Zeni L, Cassatella M A, Rossi F. Gamma interferono . 
is able to enhance the oxidative metabolism of human neutrophils. Biochem Biophys Res Commun 1986; 138: 1276-82.

26 Shalaby M R, Aggarwal B B, Rinderknecht E, Svedersky L P, Finkle B S, Palladino M A. Activation of human polymorphonuclear neutrophil functions by interferon- $\gamma$ and tumour necrosis factors. J Immunol 1985; 135: 2069-73.

27 Ferrante A, Rencis V O. Enchancement of base hexosemonophosphate shunt activity of human polymorphonuclear leucocytes by human $\beta$-interferon. Immunol Lett 1984; 8: 215-7.

28 Ferrante A, Mocatta T J. Human neutrophils require activation by mononuclear leucocyte conditioned medium to kill the pathogenic free-living amoeba, Naegleria fowleri. Clin Exp Immunol 1984; 56: 559-66.

29 Ferrante A, Abell T J. Conditioned medium from stimulated mononuclear leukocytes augments human neutrophil-mediated killing of a virulent Acanthamoeba sp. Infect Immun 1986; 51: 607-17.

30 Ferrante A, Nandoskar M, Bates E J, Goh D H B. Staphylococcus aureus-stimulated human mononuclear leucocyteconditioned medium augments the basal and stimuli-induced neutrophil respiratory burst and degranulation. Immunology 1987; 60: 431-8.

31 Ferrante A, Hill N L, Abell T J, Pruul H. Role of myeloperoxidase in the killing of Naegleria fowleri by lymphokinealtered human neutrophils. Infect Immun 1987; 55: 1047-50.

32 Ferrante A, Thong Y H. Optimal conditions for simultaneous purification of mononuclear and polymorphonuclear leucocytes from human blood by the Hypaque-Ficoll method. J Immunol Methods 1980; 36: 109-17.

33 Ferrante A, Thong Y H. Separation of mononuclear leucocytes from human blood by the one-step Hypaque-Ficoll method is dependent on blood column height. $J$ Immunol Methods 1982; 48: 81-5.

34 Janis R, Hamerman D. Articular cartilage changes in early arthritis. Bulletin of the Hospital for Joint Diseases 1969; 30: $136-52$.

35 Ugai K, Ishikawa $\mathrm{H}$, Hirohata $\mathrm{K}$, Shirane $\mathrm{H}$. Interaction of polymorphonuclear leukocytes with immune complexes trapped in rheumatoid articular cartilage. Arthritis Rheum 1983; 26: 1434-41.

36 Ishikawa H, Smiley J D, Ziff M. Electron microscopic demonstration of immunoglobulin deposition in rheumatoid cartilage. Arthritis Rheum 1975; 18: 563-76.

37 Cooke T D. Hurd E R, Ziff M, Jasin H E. The pathogenesis of chronic inflammation in experimental antigen-induced arthritis. II. Preferential localisation of antigen-antibody complexes to collagenous tissues. J Exp Med 1972; 135: 323-38.

38 Gale R, Bertouch J V, Gordon T P, Bradley J, Roberts-
Thomson $\mathrm{P}$ J. Neutrophil activation by immune complexes and the role of rheumatoid factor. Ann Rheum Dis 1984; 43: 34-9.

39 Ferrante $\mathrm{A}$, Thong $\mathrm{Y} \mathrm{H}$. Unique phagocytic process in neutrophil-mediated killing of Naegleria fowleri. Immunol Lett 1980; 2: 37-41.

40 Olsson I, Venge P. The role of the human neutrophil in the inflammatory reaction. Allergy 1980; 35: 1-13.

41 Dewald B, Bretz U, Baggiolini M. Release of gelatinase from a novel secretory compartment of human neutrophils. J Clin Invest 1982; 70: 518-25.

42 Campbell E J, Senior R M, McDonald J A, Cox D L. Proteolysis by neutrophils. Relative importance of cell-substrate contact and oxidative inactivation of proteinase inhibitors in vitro. $J$ Clin Invest 1982; 70: 845-52.

43 Ohlsson K. $\alpha_{1}$-Antitrypsin and $\alpha_{2}$-macroglobulin interactions with human neutrophil collagenase and elastase. Ann NY Acad Sci 1975; 256: 409-19.

44 Rindler-Ludwig $\mathbf{R}$, Braunsteiner $\mathrm{H}$. Cationic proteins from human neutrophil granulocytes. Evidence for their chymotrypsin-like properties. Biochim Biophys Acta 1975; 379: 606-17.

45 Werb Z, Dingle J T, Reynolds J J, Barrett A J. Proteoglycandegrading enzymes of rabbit fibroblasts and granulocytes. Biochem J 1978; 173: 949-58.

46 Fantone J C, Ward P A. Role of oxygen-derived free radicals and metabolites in leukocyte-dependent inflammatory reactions. Am J Pathol 1982; 107: 396-418.

47 Bates E J, Harper G S, Lowther D A, Preston B N. Effect of oxygen-derived reactive species on cartilage proteoglycanhyaluronate aggregates. Biochem Int 1984; 8: 629-37.

48 Herman J H, Khosla R C, Mowery C S, Appel A M. Modulation of chondrocyte synthesis by lymphokine-rich conditioned media. Arthritis Rheum 1982; 25: 668-76.

49 Tyler J A. Articular cartilage cultured with catabolin (pig interleukin 1) synthesise a decreased number of normal proteoglycan molecules. Biochem J 1985; 227: 869-78.

50 Krakauer T, Oppenheim J J, Jasin H E. Human interleukin 1 mediates cartilage matrix degradation. Cell Immunol 1985; 91: 92-9.

51 Pettipher E R, Higgs G A, Henderson B. Arthritogenic activity of interleukin 1. Agents Actions 1986; 19: 337-8.

52 Herman J H, Musgrave D S, Dennis M V. Phytomitogeninduced, lymphokine-mediated cartilage proteoglycan degradation. Arthritis Rheum 1977; 20: 922-32.

53 Jasin H E, Dingle J T. Human mononuclear cell factors mediate cartilage matrix degradation through chondrocyte activation. $J$ Clin Invest 1981; 68: 571-81. 